\title{
$\dot{\nabla}_{\text {Nursing }}^{\text {Global Academic }}$
}

ARTIGO DE REVISÃO

\section{Panorama das publicações em saúde mental no contexto da pandemia por COVID-19: scoping review}

\author{
Overview of publications on mental health in the context of the COVID-19 pandemic: scoping review
}

Resumen de publicaciones sobre salud mental en el contexto de la pandemia COVID-19: revisión de alcance

\author{
Rodrigo Jácob Moreira de \\ Freitas $^{1}$ \\ ORCID: 0000-0002-5528-2995 \\ Natana Abreu de Moura² \\ ORCID: 0000-0002-2517-9946 \\ Liane Araújo Teixeira ${ }^{2}$ \\ ORCID: 0000-0002-5105-7571 \\ Ana Paula Nunes de Lima \\ Fernandes ${ }^{3}$ \\ ORCID: 0000-0002-0503-3333 \\ Ana Ruth Macêdo Monteiro ${ }^{2}$ \\ ORCID: 0000-0002-1130-1293 \\ ${ }^{1}$ Universidade do Estado do Rio \\ Grande do Norte. Rio Grande do \\ Norte, Brasil. \\ ${ }^{2}$ Universidade Estadual do Ceará. \\ Ceará, Brasil. \\ ${ }^{3}$ Universidade Federal do Rio \\ Grande do Norte. Rio Grande do \\ Norte, Brasil.
}

\section{Como citar este artigo:}

Freitas RJM, Moura NA, Teixeira LA, Fernandes APNL, Monteiro ARM. Panorama das publicações em saúde mental no contexto da pandemia por COVID-19: scoping review. Glob Acad Nurs. 2021;2(1):e84. https://dx.doi.org/10.5935/26755602.20200084

Autor correspondente: Rodrigo Jácob Moreira de Freitas E-mail: rodrigojmf@gmail.com

Editor Chefe: Caroliny dos Santos Guimarães da Fonseca Editor Executivo: Kátia dos Santos Armada de Oliveira

Submissão: 02-12-2020

Aprovação: 16-12-2020

\section{Resumo}

Objetivou-se mapear a literatura científica sobre a saúde mental da população no contexto da pandemia de COVID-19. Scoping review realizada através da busca em seis bases de dados eletrônicas, buscadores eletrônicos e por meio das referências dos artigos selecionados. Foi incluído artigos completos, nos idiomas português, inglês ou espanhol sobre saúde mental no contexto da pandemia de COVID-19. Excluiu-se artigos com publicação há mais de 1 ano por não haver relação com o início da pandemia. Mapeou-se 48 estudos publicados no ano de 2020 (100\%), 45 (93,75\%) em inglês, 27 (56,25\%) foi proveniente da China; 30 (62,5\%) eram estudos transversais; $30(62,5 \%)$ tinham o nível de evidência IV. Os dados foram organizados em três temáticas: Saúde mental da população geral e grupos vulneráveis relacionados com os impactos da pandemia; Saúde mental dos profissionais de saúde; Estratégias de saúde mental diante da pandemia por COVID-19. O conhecimento dos impactos na saúde mental da população durante a pandemia por COVID-19 exige estratégias para enfrentamento durante e após pandemia. Sugere-se pesquisas colaborativas, multicêntricas e interdisciplinares a fim de aprofundar a compreensão das implicações para a saúde da crise atual.

Descritores: Saúde Mental; Assistência à Saúde Mental; Vírus da SARS; Infecção por Coronavírus; Pandemias.

Abstract

The aim was to map the scientific literature on the mental health of the population in the context of the COVID-19 pandemic. Scoping review carried out by searching six electronic databases, electronic search engines and through the references of selected articles. Complete articles were included, in Portuguese, English or Spanish on mental health in the context of the COVID-19 pandemic. Articles that were published more than 1 year ago were excluded because there was no relationship with the beginning of the pandemic. 48 studies published in the year 2020 (100\%) were mapped, 45 (93.75\%) in English, 27 (56.25\%) were from China; 30 (62.5\%) were cross-sectional studies; 30 (62.5\%) had evidence level IV. The data were organized into three themes: Mental health of the general population and vulnerable groups related to the impacts of the pandemic; Mental health of health professionals; Mental health strategies in the face of the COVID-19 pandemic. Knowledge of the mental health impacts of the population during the COVID-19 pandemic requires strategies for coping during and after the pandemic. Collaborative, multicenter, and interdisciplinary research is suggested to deepen the understanding of the health implications of the current crisis.

Descriptors: Mental Health; Mental Health Assistance; SARS Virus; Infection from Coronavirus; Pandemic.

\section{Resumén}

El objetivo fue mapear la literatura científica sobre la salud mental de la población en el contexto de la pandemia COVID-19. Revisión del alcance realizada mediante la búsqueda en seis bases de datos electrónicas, motores de búsqueda electrónicos y a través de las referencias de los artículos seleccionados. Se incluyeron artículos completos en portugués, inglés o español sobre salud mental en el contexto de la pandemia COVID-19. Se excluyeron los artículos que se publicaron hace más de 1 año porque no tenían relación con el inicio de la pandemia. Se mapearon 48 estudios publicados en el año 2020 (100\%), 45 $(93,75 \%)$ en inglés, 27 (56,25\%) fueron de China; 30 (62,5\%) fueron estudios transversales; $30(62,5 \%)$ tenían nivel de evidencia IV. Los datos se organizaron en tres temas: salud mental de la población en general y grupos vulnerables relacionados con los impactos de la pandemia; Salud mental de los profesionales de la salud; Estrategias de salud mental ante la pandemia COVID-19. La conciencia de los impactos en la salud mental de la población durante la pandemia COVID-19 requiere estrategias para hacer frente durante y después de la pandemia. Se sugiere la investigación colaborativa, multicéntrica e interdisciplinaria con el fin de profundizar en la comprensión de las implicaciones para la salud de la crisis actual.

Descriptores: Salud Mental; Asistencia de Salud Mental; Virus del SARS; Infección por Coronavirus; Pandemias. 


\section{Introdução}

Em dezembro de 2019 um conjunto de casos de pneumonia de origem desconhecida ocorreu em Wuhan, China, causando preocupação entre as autoridades de saúde. Depois de descartadas outras potenciais causas, um novo patógeno foi descoberto e identificado, o vírus SARSCoV-2, denominado pela Organização Mundial da Saúde (OMS) responsável pela COVID-19. ${ }^{1}$

Desde sua recente descoberta, aconteceu uma rápida disseminação do vírus, declarando a OMS uma pandemia em março de 2020, por causa dos mais de 27 países que confirmaram ter casos de COVID-19, incluindo o Brasil. O aumento dos números de casos, principalmente, o aumento do número de óbitos se deve pelo fato do vírus causar dificuldade respiratória aguda grave, além de ocasionar infecções que podem levar a pneumonia viral. ${ }^{2}$ Diante do alto poder de transmissão e da falta de tratamentos com eficácia estabelecida, as recomendações dos órgãos de saúde é que temporariamente todos permaneçam em quarentena ou isolamento social.

Adotado como principal estratégia no plano de contingência, o isolamento social consiste no funcionamento apenas de serviços essenciais para sobrevivência humana em sociedade, como hospitais, supermercados, farmácias, dentre outros, permanecendo todo o resto da população, principalmente idosos, portadores de doenças crônicas e crianças em casa. ${ }^{2}$

Essa estratégia de diminuição da disseminação do vírus tem gerado muitas especulações acerca dos seus impactos, que variam desde os impactos sobre a economia, o sustento de milhares de famílias, até os sentimentos que tem surgido devido ao isolamento em residências, a falta de contato social, preocupação com familiares, a sobrecarga dos profissionais de saúde, e a solidão dos que estão contaminados pela COVID-19. Não somente pelo isolamento, mas as pessoas acometidas direta ou indiretamente pela doença podem sofrer consequências em sua saúde mental, o que se leva a pensar que o contexto da pandemia vem afetar a saúde mental de todas as pessoas, em um maior ou menor grau.

Com isso, para se compreender as repercussões psicológicas de uma pandemia, as emoções envolvidas, como medo, raiva, angústia, devem ser analisadas. Essas emoções surgem em resposta ao evento potencialmente ameaçador para a existência, que nesse caso é a pandemia, entretanto, ao se manifestar de forma crônica ou desproporcional, se torna prejudicial e pode ser um componente essencial no desenvolvimento de transtornos psiquiátricos. $^{3}$

O medo aumenta os níveis de ansiedade e estresse em indivíduos saudáveis além de intensificar os sintomas de quem tem transtorno psiquiátrico. Assim, o estudo justificase, pois, se faz importante investigar acerca da saúde mental das pessoas no período da pandemia, visto que o número de pessoas cuja saúde mental é afetada durante esse período é maior do que o número de pessoas infectadas pela COVID19. As implicações acerca do prejuízo na saúde mental das pessoas podem ter uma duração maior que a pandemia e ter impactos psicossociais e econômicos incalculáveis se considerarem os diferentes contextos em que repercute. ${ }^{3}$

Deste modo, no intuito de conhecer e avaliar as implicações diretas no cotidiano e na saúde mental da população é necessário se questionar sobre os efeitos psicológicos da pandemia. Através da estratégia PCC (população, conceito e contexto) a pergunta de pesquisa foi formulada: O que se sabe na literatura sobre a saúde mental da população em geral no contexto da COVID-19? Assim, objetiva-se mapear a literatura científica sobre a saúde mental da população no contexto da pandemia de COVID19.

\section{Metodologia}

Trata-se de uma Scoping Review (SR), de caráter exploratório e abordagem qualitativa, com foco na saúde mental dos indivíduos no contexto da pandemia da COVID19. Todos os métodos de revisão existentes são úteis para cada situação específica, pois oferecem um conjunto de ferramentas para abordagem da literatura.

Neste estudo, optou-se pela SR por tratar-se de um processo de mapeamento dos conceitos-chave que sustentam uma área de investigação e as principais fontes de conhecimento disponíveis.

O mapeamento se refere à sumarização das informações que permite a identificação de evidências que transmitem conhecimento amplo e profundo de determinado objeto. ${ }^{4}$ Por tratar-se de um fenômeno novo, torna-se necessário o mapeamento e clarificação das relações entre saúde mental no contexto da pandemia por COVID-19.

Para realização da SR a priori foi construído um protocolo de pesquisa que norteou a construção de todos as etapas. Foram seguidos os seguintes passos: identificação da questão e pesquisa, identificação dos estudos relevantes, seleção dos estudos, mapeamento dos dados e coleta, resumo e relato dos resultados. ${ }^{4}$

A primeira etapa foi realizada a partir da construção da questão de pesquisa e definição da População - Conceito - Contexto (PCC) em que o estudo seria focado. Como população $(P)$ foram elencadas: populações em geral, como crianças, adultos, idosos e profissionais de saúde. O conceito (C) trabalhado foi o de saúde mental e o contexto (C) foi a pandemia da COVID-19. Partiu-se da seguinte questão: O que se sabe na literatura sobre a saúde mental da população em geral no contexto da COVID-19?

Para a busca foi adotada a estratégia preconizada pelo manual para Scoping Review construído pelo Instituto Joanna Briggs. ${ }^{4}$ Iniciou-se por meio de uma ampla busca em bases de dados utilizando descritores controlados, posteriormente foi realizada a verificação em buscador eletrônico e por último, uma busca reversa nas referências.

As bases de dados utilizadas foram: Cochrane, Psycinfo, LILACS, Scopus, Pubmed e Web of Science. Para a obtenção dos artigos, a busca foi realizada por pares, em um mesmo horário e em dispositivos separados a partir dos descritores controlados indexados no Descritores em Ciências da Saúde (DeCS) e Medical Subject Headings (MeSH). 
Foram utilizados os seguintes descritores do DeCS: 1\# (Saúde Mental; Salud Mental; Mental Health), 2\# (Vírus da SARS; Virus del SRAS; SARS Virus), 3\# (Síndrome Respiratória Aguda Grave; Síndrome Respiratorio Agudo Grave; Severe Acute Respiratory Syndrome). Utilizou-se também os descritores MeSH: \#1 (Mental Health), \#2 (SARS Virus), \#3 (Coronavirus Infections) e \#4 (Pandemics).

Foram utilizados os cruzamentos \#1 AND \#2 e \#1 AND \#3, nas bases que utilizam os descritores DeCS. Para as que se utilizam dos descritores $\mathrm{MeSH}$ foram utilizados os cruzamentos: \#1 AND \#2 OR \#3 OR \#4; \#1 AND \#3 AND \#4. Para a verificação nos buscadores eletrônicos "Google Scholar" e "Science Direct", foram utilizadas as palavraschave "COVID-19" e "Mental Health". As buscas ocorreram entre os meses de abril e maio de 2020.

Após a realização da busca, foi iniciado o processo de elegibilidade por meio dos critérios de inclusão e exclusão. Foram adotados como critérios de inclusão: estudos que tenham sido desenvolvidos com a população em geral (crianças, adultos, idosos, profissionais de saúde) no contexto da pandemia da COVID-19; estudos que abordem a saúde mental; estudos que foram realizados no contexto da pandemia de COVID-19; artigos completos disponíveis nas bases de dados selecionadas; artigos disponíveis nos idiomas Português, Inglês ou Espanhol; estudos que tenham sido finalizados e estejam publicados ou em preprints. Como critérios de exclusão foram elencados: editoriais; cartas ao editor; comentários; resumos; artigos que não abordem a temática relevante ao alcance do objetivo da revisão; artigos com publicação há mais de 1 ano.

O método recomenda que as informações da literatura cinzenta sejam agregadas no estudo, a fim de mapear o que existe disponível pela temática, por este motivo optou-se pela inclusão de artigos preprints. Porém, destaca-se que entrou na revisão apenas artigos com avaliação peer review e aprovado pelos periódicos, excluindo-se artigos apenas em submissão.

Cada um dos artigos passou por uma avaliação da qualidade metodológica, para cada um dos delineamentos foi utilizado um instrumento de avaliação ou de recomendação para redação. Para os estudos observacionais foi utilizado o Strengthening the Reporting of Observational Studies in Epidemiology (STROBE), para os estudos experimentais o Consolidated Standards of Reporting Trials (CONSORT), para os estudos qualitativos o Consolidated Criteria for Reporting Qualitative Studies (COREQ) e para as revisões o Preferred Reporting Items for Systematic Reviews and Meta-Analyses (PRISMA). Após a avaliação e aprovação obtida por pares, o estudo era integrado a amostra. 0 recorte temporal foi utilizado para que os estudos tenham relação com o início da circulação do vírus.

Após a seleção da amostra, foi realizada a busca por meio das referências dos artigos selecionados, porém não houve a inclusão de nenhum novo artigo.

Para a extração dos dados, foi elaborado um instrumento para auxiliar no mapeamento, contendo informações relacionadas a identificação da publicação (título, autores, país, ano de publicação e idioma), aspectos metodológicos e do conteúdo do estudo (objetivo da investigação, metodologia, tipo de abordagem, população e amostra, além das principais descobertas do estudo) e aspectos da saúde mental das pessoas no contexto da pandemia da COVID-19. Os dados foram extraídos, mapeados, sintetizados e organizados por temáticas. O passo a passo do quantitativo identificado apresenta-se disponível na Figura 1.

A pesquisa foi realizada seguindo os princípios éticos conforme a Resolução n. 466, de dezembro de 2012. Por se tratar de uma SR, dispensa o envio para o Comitê de Ética em Pesquisa (CEP).

\section{Resultados}

Através das buscas nas bases de dados mapeou-se 48 estudos científicos que discutiram sobre a temática da saúde mental em meio a pandemia do vírus SARS-CoV-2, conforme Figura 1.

As produções foram publicadas no ano de 2020 (100\%), o que pode ser explicado pela a Organização Mundial da Saúde ter caracterizado a disseminação do Coronavírus como pandemia apenas em março do ano corrente, o idioma inglês foi o predominante $(93,75 \%)$, o país de maior autoria foi a China $(56,25 \%)$, epicentro inicial da COVID19; os estudos primários (62,5\%), com destaque para o delineamento metodológico quantitativo (transversais) foram os mais relatados, em relação aos tipos de publicação, a maioria (98\%) são artigos (paper), encontrado apenas um manual; a maior parte dos estudos foram localizados através do buscador acadêmico "Google acadêmico" (50\%); o nível de evidência das pesquisas se encontram em IV (62,5\%) e VI $(37,5)$. Os detalhamentos dos dados seguem na Tabela 1.

De acordo com o enfoque dos trabalhos, foram organizados em três temáticas principais: Saúde mental da população geral e grupos vulneráveis relacionados com os impactos da pandemia; Estratégias de saúde mental diante da pandemia por COVID-19; Saúde mental dos profissionais de saúde.

\section{Saúde mental da população geral e grupos vulneráveis relacionados com os impactos da pandemia}

Identificou-se Transtorno de Estresse Póstraumático (TEPT), emoções negativas (confusão, raiva e estresse), transtornos de ansiedade, depressão, compulsão obsessiva, distúrbios do sono, aumentos do consumo de álcool, maior preocupação com familiares e a saúde. ${ }^{5-16}$ Mudanças de comportamento durante a pandemia de COVID-19 em relação a saúde também foram relatadas. Informações claras sobre prevenção e contágio preveniram problemas de saúde mental. ${ }^{17-18}$ Sentimentos positivos em relação ao apoio familiar e sentimentos negativos devido a pandemia e exposição a mídia. ${ }^{19-22}$

Aponta-se o aumento da ansiedade em populações específicas (estudantes) e piora do quadro de crianças com Transtorno de Hiperatividade e Déficit de Atenção (TDAH). ${ }^{23-}$ 26 Há a necessidade de apoio das instituições aos trabalhadores, visto que piores condições de saúde física, mental e angústia foram maiores naqueles que pararam de 
trabalhar. ${ }^{27-29}$ É urgente a necessidade de cuidado em saúde mental durante e após o fim da pandemia. ${ }^{30-38}$

\section{Estratégias de saúde mental diante da pandemia por COVID-19}

Aponta-se ações governamentais para emergências em crises psicológicas além do uso de teorias para compreender o vivido e o que esperar no período de pós isolamento social. ${ }^{42-43}$ É imperativo que cada indivíduo cuide de sua saúde física e mental. Alguns estudos relatam os benefícios da companhia de animais na ajuda para aliviar estressores físicos, emocionais e psicológicos. ${ }^{44-49}$ É importante a solidariedade em vez de estigma ou discriminação para conter a propagação do Coronavírus.
Deve-se identificar grupos vulneráveis e suas demandas, oferecendo informação aberta e transparente sobre o surto e a doença ao público global. ${ }^{50-52}$

\section{Saúde mental dos profissionais de saúde}

Destaca-se o impacto na saúde mental dos profissionais de saúde. A crise de saúde atual afeta o eu e a identidade das pessoas que são confrontadas com a discrepância entre suas necessidades psicológicas usuais e as realidades atuais. ${ }^{39-40}$ Crianças, horas de trabalho por semana e ansiedade foram os principais fatores que afetam o estresse do enfermeiro. ${ }^{41}$

Figura 1. Fluxograma de elegibilidade dos estudos, PRISMA ScR. Fortaleza, CE, Brasil, 2020

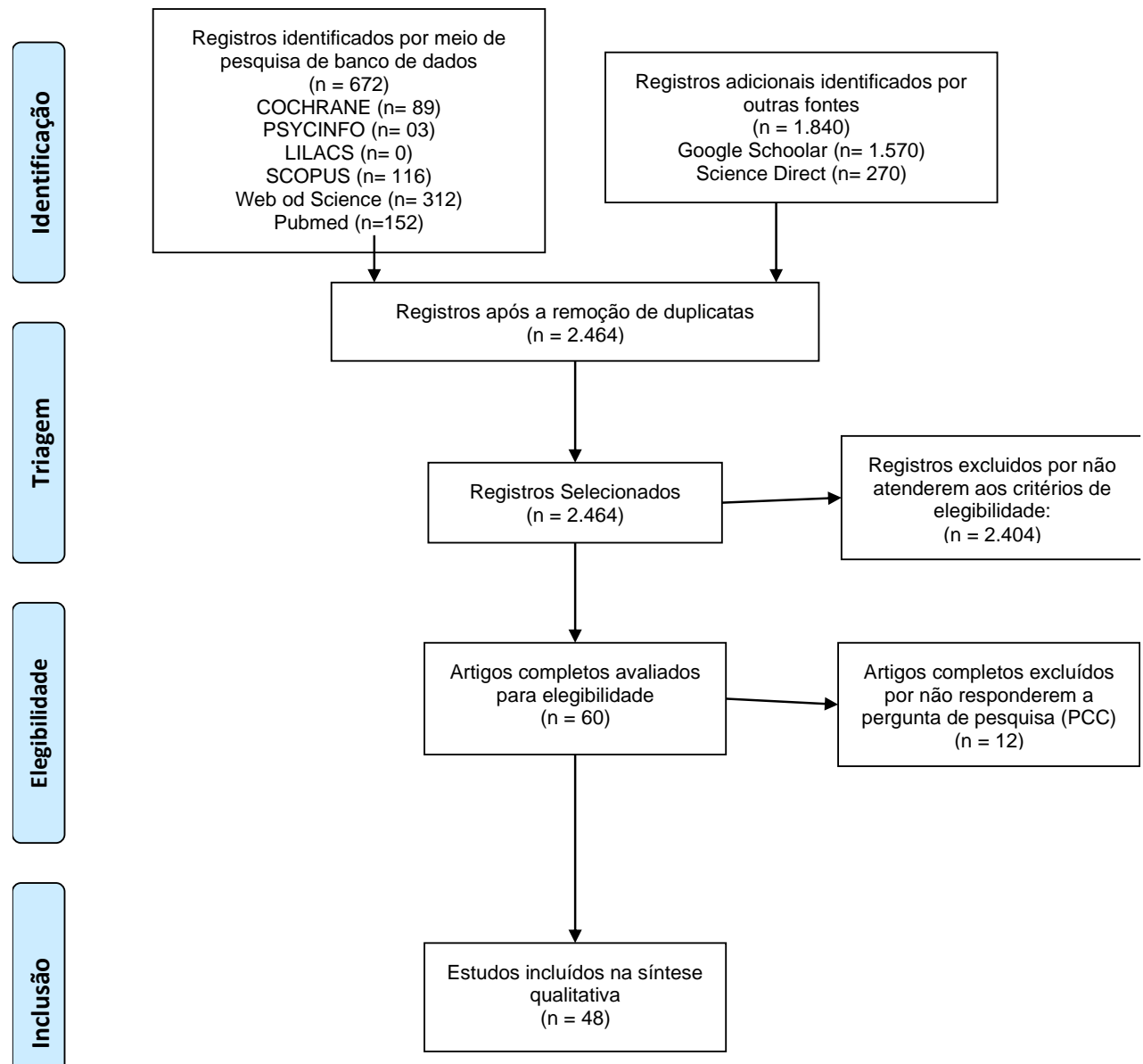

Tabela 1. Caracterização dos estudos quanto ao idioma, ano de publicação, local do estudo, delineamento metodológico/amostra, busca online. Fortaleza, CE, Brasil, $2020(n=48)$

\begin{tabular}{lc}
\hline Variáveis & $\mathbf{n}(\%)$ \\
\hline Idioma & \\
Inglês & $45(93,75)$ \\
Português & $2(4,17)$ \\
Espanhol & $1(2,08)$ \\
Local do estudo & \\
\hline
\end{tabular}




\begin{tabular}{lc}
\hline China & $27(56,25)$ \\
EUA & $4(8,33)$ \\
Europa (Reino Unido, Dinamarca, França) & $5(10,42)$ \\
Índia & $3(6,25)$ \\
Parceria entre países (Itália, Paraguai e Brasili Singapura, Índia e EUA; Itália e Canadá) & $3(6,25)$ \\
Outros (Brasil, Peru, Singapura, Irã e Vietnã) & $6(12,5)$ \\
Delineamento metodológico/amostra & \\
Estudos primários & $30(62,5)$ \\
Revisão da Literatura & $9(18,75)$ \\
Estudos de reflexão/teórico & $6(12,5)$ \\
Relatos de experiência & $2(4,17)$ \\
Manual & $1(2,08)$ \\
Busca on-line & \\
Google Scholar & $24(50)$ \\
Scopus & $14(29,17)$ \\
Science Direct & $9(18,75)$ \\
PubMed & $1(2,08)$ \\
\hline
\end{tabular}

\section{Discussão}

As alterações psicológicas podem afetar indivíduos que tiveram ou não COVID-19. A presença de transtorno de estresse pós-traumático, principalmente após o início do surto na $\mathrm{China}^{5}$ e a sua associação com sentimentos negativos, como raiva e indignação foram evidenciados nos estudos. ${ }^{6,7}$

Ansiedade e depressão foram bastante relatadas nas publicações, foi detectado níveis moderados a graves desses dois eventos psicológicos durante a situação de pandemia, ${ }^{9,18,21}$ sintomas que podem perdurar e se tornarem crônicos, ${ }^{6}$ houve associação entre álcool, ansiedade, depressão com a diminuição do bem-estar mental, ${ }^{8}$ os estressores que tem levado a esses sintomas podem ser tidos como o tempo de quarentena que pode se prolongar, o medo de contrair a doença, entre outros, como distúrbios do sono e ter ou já ter tido a doença. ${ }^{6,11,12}$

O risco de ansiedade foi mais visto em mulheres e pessoas acima dos 40 anos, ${ }^{14,22}$ em contraponto encontrouse Transtorno de Ansiedade Generalizado e sintomas depressivos mais em jovens do que em idosos. ${ }^{12} \mathrm{Em}$ relação a saúde mental também foram vistos os fatores de proteção, como atitudes positivas, das pessoas cumprirem as diretrizes governamentais de distanciamento social e higienização, ${ }^{17}$ além de terem informações seguras sobre como cuidar da saúde, o grau de confiabilidade na equipe de saúde e sobre as chances de contrair o vírus, ${ }^{9}$ outro ponto positivo foi o fato de apesar da distância poderem se sentir amparados por familiares e amigos. ${ }^{17}$

O papel das mídias sociais foi tido como fator estressante por exagerarem na divulgação das notícias e na situação da pandemia, podendo aumentar a sensação de medo e angústia, além de paranoia e distúrbios do sono. ${ }^{17,20,21}$

Os estudos abordaram as repercussões da pandemia no estado psicológico de estudantes, crianças e adultos que já sofriam de alguns transtornos mentais, pessoas em confinamento por estarem infectadas, aquelas que continuaram trabalhando de forma remota e aqueles que tiveram que procurar assistência psicológica devido a disseminação do SARS-Cov-2.

Nos estudantes universitários foram encontrados estados de ansiedade grave e estresse psicológico, principalmente por saberem de parentes ou conhecidos que contraíram COVID-19, como fatores positivos de proteção à saúde mental relatou-se não terem problemas financeiros, morarem com os pais e em áreas urbanas. ${ }^{23,26}$

Crianças com transtorno de déficit de atenção e hiperatividade foram avaliadas através de escalar por seus pais, estes identificaram piora no quadro das mesmas durante a pandemia. ${ }^{25} \mathrm{Em}$ Hospital Psiquiátrico na China foi visto vários desafios, como continuar o tratamento de pacientes que viviam na comunidade, por conta da limitação do transporte público, ter que controlar e prevenir a disseminação do Coronavírus dentro do Hospital e o próprio esgotamento do serviço. ${ }^{24}$

Foi identificado que as pessoas que continuaram trabalhando remotamente em casa precisavam de manutenção dos laços sociais para poderem manter sua produtividade a longo prazo, enquanto pessoas que pararam de trabalhar tiveram piores resultados de saúde física e emocional. 27,28

O trabalho psicológico com pessoas internadas e infectadas com COVID-19 se deu no âmbito de fortalecimento emocional e de informações recebidas, identificou que pessoas com a doença tinham maiores níveis de depressão e menor qualidade de saúde. A ansiedade e o estresse foram bem associados a baixa qualidade do sono, mesmo na presença de um bom capital social, em pessoas com Coronavírus que se isolaram durante os 14 dias, evidenciando os abalos na saúde mental devido a pandemia. ${ }^{29,16}$

Os artigos ${ }^{31-33,35-39,37-38}$ apontam os profissionais de saúde como os principais sujeitos afetados mentalmente durante a pandemia. Os profissionais de saúde têm o dever de cuidar dos pacientes infectados, estreitar o contato com 
a família dos pacientes e, algumas vezes, enfrentar o inquérito público. Profissionais de saúde da linha de frente, especialmente aqueles em Wuhan, têm contato próximo com pacientes infectados, carga de trabalho excessiva, isolamento e discriminação são frequentemente relatados e, portanto, são altamente vulneráveis a sofrer exaustão física, medo, distúrbios emocionais e problemas de sono. ${ }^{42}$

Isso vai de encontro com a revisão da literatura ${ }^{35,39}$ sobre os aspectos psicológicos em epidemias anteriores, como de SARS, MERS, influenza e Ebola, onde os indivíduos que foram infectados e os profissionais de saúde foram os mais afetados psicologicamente.

No artigo $^{32} 5,3 \%(\mathrm{~N}=48)$ apresentaram resultado positivo para depressão moderada a muito grave, $8,7 \%$ $(\mathrm{N}=79)$ para ansiedade moderada a extremamente grave, $2,2 \%(\mathrm{~N}=20)$ para estresse moderado a extremamente grave e $3,8 \%(N=34)$ para níveis moderado a grave de sofrimento psicológico.

Os sintomas mais comuns relatados pelos profissionais foram dor de cabeça $(289,31,9 \%)$, dor de garganta $(\mathrm{N}=304,33,6 \%)$, ansiedade $(\mathrm{N}=242,26,7 \%)$, letargia ( $N=241,26,6 \%$ ) e insônia ( $N=190,21,0 \%)$. O mesmo artigo apontou a associação positivas desses sintomas a problemas de saúde mental como a depressão, ansiedade, estresse e Transtorno de Estresse Pós-traumático (TEPT). Resultados semelhantes foram obtidos nos artigos ${ }^{31,36-38}$ onde depressão, estresse, medo, ansiedade, insônia, angústia, sintomas obsessivo-compulsivos e somatizações foram apontados.

Pode-se destacar ainda o sofrimento psicológico resultante de ações, ou a falta delas, que violam o código moral ou ético profissional, pensamentos negativos sobre si mesmos ou sobre os outros (por exemplo, "eu sou uma pessoa terrível" ou "meus chefes não se importam com a vida das pessoas"), bem como sentimentos intensos de vergonha, culpa, ou nojo, e até ideação suicida. ${ }^{30}$

Dos profissionais de saúde, a equipe médica que atua nos setores respiratórios, de emergência, UTI, setor de doenças infecciosas teve duas vezes mais probabilidade de sofrer de ansiedade e depressão do que os funcionários administrativos com pouca possibilidade de contato com pacientes COVID-19 positivo. ${ }^{37}$ As razões podem estar relacionadas a sobrecarga com situações que têm relevância pessoal, como cuidar de alguém que os lembre de um parente ou amigo, ${ }^{30}$ as muitas dificuldades de estar seguro no trabalho, o insuficiente entendimento do vírus, a falta de conhecimento de prevenção e controle, a carga de trabalho a longo prazo, o alto risco de exposição a pacientes com COVID-19, a escassez de equipamentos de proteção médica, a falta de descanso e a exposição a eventos críticos da vida, como a morte. ${ }^{36}$

Além disso, o perfil de profissionais com mais possibilidade de adoecimento mental foram vistos em enfermeiros, mulheres que trabalham na linha de frente da COVID-19. ${ }^{31}$ Esse acometimento pode estar associado ao horário de trabalho por semana e a carga de estresse dos enfermeiros; ;1 a escassez de equipamentos de proteção, resultando em fadiga e desconforto; deixar de atender às necessidades físicas e psicológicas dos pacientes trouxe uma sensação de desamparo; medo, por trabalharem no setor especifico para COVID-19; preocupação com os pacientes em um ambiente isolado, com poucos cuidadores e muitos pacientes; preocupação com o impacto do surto na saúde de suas famílias. ${ }^{33}$

Nesse mesmo estudo, apontam que os enfermeiros desenvolveram mecanismos de defesa psicológica, como especulação, isolamento, depressão, distração, autoconsciência, humor, racionalização etc. Como fatores de proteção foram elencados a ajuda e apoio entre os membros da equipe, enfermeiros sentiram a boa vontade, o respeito, a cooperação ativa e a gratidão do paciente, e o apoio da família.

Embora a maioria dos enfermeiros tenha emoções negativas, como medo, ansiedade e preocupação, as emoções positivas foram aparecendo de forma progressiva, à medida que os fatores de proteção iam sendo fortalecidos. Os enfermeiros também avaliaram o progresso da prevenção e controle da epidemia e sentiram confiança na capacidade médica do governo. ${ }^{33}$

A identificação de problemas e o suporte precoce são essenciais. É preciso preparar adequadamente a equipe para o trabalho e os desafios associados, reduzindo o risco de problemas de saúde mental. Além disso, o apoio dos colegas de equipe e gerente, o suporte aos gestores, evitar falar sobre culpa e vergonha e se concentrar em outros estressores durante a terapia, podem proteger os profissionais dos problemas de saúde mental. ${ }^{30}$

Esses recursos são vistos como importantes para aliviar distúrbios agudos da saúde mental e melhorar suas percepções de saúde física. É preciso um investimento maior nas ferramentas de saúde mental, dos médicos, da sociedade, e um arsenal para proteger e cuidar de futuras equipes médicas e de enfermagem que inesperadamente estão nas linhas de frente perigosas da resposta a doenças. ${ }^{38}$

Os artigos ${ }^{42-43,49}$ apontam que são necessárias estratégias globais para lidar com os problemas de saúde mental relacionados a esse contexto. As estratégias de saúde mental abordadas são em sua maioria da China, visto que é possível prever mais ou menos as consequências esperadas na saúde mental e física das partes mais vulneráveis da população a partir das experiências de epidemias anteriores vividas por esse país.

No artigo ${ }^{45}$, em relatório publicado no país, destaca a importância de liberar atualizações oficiais substanciais em intervalos regulares durante um evento de crise e monitorar as mídias sociais para reduzir a exposição a informações enganosas. $\mathrm{O}$ apoio e o acompanhamento da saúde mental devem ser fornecidos até 6 meses após a liberação do isolamento para os indivíduos com status de saúde mental vulnerável anterior. $O$ apoio deve incluir informações precisas, bem como suprimentos adequados para os sujeitos, incluindo alimentos, roupas e acomodações, se necessário.

As ações por parte do governo chinês incluíram a compreensão do estado de saúde mental em diferentes populações influenciadas pelo surto de COVID-19, a identificação de pessoas com alto risco de suicídio e agressão 
e fornecimento de intervenções psicológicas apropriadas para os necessitados. ${ }^{42}$

Dessa forma, foi elaborado um documento intitulado "Diretrizes para a Linha Direta de Assistência Psicológica durante a Prevenção e Controle da Nova Pneumonia por Coronavírus" para psiquiatras em hospitais psiquiátricos e departamentos psicológicos em hospitais gerais poderem garantir assistência psicológica em resposta ao surto. ${ }^{42,44}$

As diretrizes devem ser implementadas sob a orientação de profissionais treinados em saúde mental e adotar intervenções emergenciais de crise psicológica, aconselhamento psicológico e estabelecer grupos de especialistas em assistência psicológica para fornecer orientação profissional e coordenar com as autoridades de saúde. ${ }^{44}$

Devido a diferentes populações serem afetadas pela epidemia em diferentes graus, os psiquiatras identificaram populações de alto risco para priorizar seus esforços, a fim de evitar eventos extremos, como suicídio e comportamentos impulsivos. Da população geral, foram priorizadas pacientes infectados e doentes, suas famílias e colegas; indivíduos e suas relações com a comunidade; indivíduos com condições médicas preexistentes (físicas e / ou físicas); profissionais de saúde, especialmente enfermeiros e médicos que trabalham diretamente com pessoas doentes ou em quarentena. ${ }^{44,45}$

A partir disso, foi estabelecido grupos-alvo principais e prioridades de acordo com critérios: 1) Pacientes com sintomas graves de COVID-19, equipe médica da linha de frente, pesquisadores ou equipe administrativa; 2) Pacientes com sintomas leves de COVID-19, contatos próximos, pacientes suspeitos ou pacientes com febre que chegam ao hospital para tratamento; 3) Pessoas relacionadas ao primeiro e à população de segundo nível, como familiares, colegas ou amigos; socorristas, comandantes, funcionários administrativos ou voluntários; 4) Pessoas em áreas afetadas, grupos suscetíveis ou público em geral. ${ }^{51}$

A Comissão Nacional de Saúde da China (NHC) integrou a Intervenção de Crise Psicológica (ICP) às ações de prevenção de doenças. O objetivo do ICP é minimizar os danos psicológicos e fornecer assistência oportuna à prevenção e controle da epidemia. A implementação deve seguir diretrizes rigorosas, a fim de reduzir a exposição das pessoas ao estresse e traumas. A ICP fornece serviços no local para populações de primeiro e segundo nível e suporte psicológico remoto e em tempo real (telefone e internet) para populações de terceiro e quarto níveis. ${ }^{42,44}$

Para o atendimento remoto, foi disponibilizado uma linha direta gratuita de 24 horas, 7 dias por semana, criada para que o público obtivesse respostas para as perguntas e preocupações, revivesse as preocupações e o pânico e eliminasse os boatos ou informações erradas. ${ }^{50}$ Esses serviços de linha direta deveriam ser estabelecidos por instituições psiquiátricas, centros de aconselhamento e educação em saúde mental dos estudantes em universidades, associações de saúde mental e sociedades acadêmicas. $^{42}$
Além disso, foi criado artigos e vídeos educacionais on-line para serem divulgados ao público; ajuda psicológica para lidar com a COVID-19 para populações específicas, incluindo idosos, crianças e adolescentes, gestantes e profissionais de saúde; vídeos de educação em saúde mental para o público em geral através do WeChat e de outras plataformas da Internet na fase inicial do surto. ${ }^{42}$

Em nível de ações por parte dos profissionais de saúde, o apoio psicológico requer o estabelecimento de equipes multidisciplinares de saúde mental, comunicação clara com atualizações regulares e precisas sobre o SARSCoV-2, planos de surto e tratamento e uso de dispositivos e aplicativos eletrônicos profissionais para evite contato próximo entre si. ${ }^{51}$

De um modo geral essas ações incluíam medidas para prevenir os riscos psicológicos inerentes ao confinamento: manutenção de sono regular, exercício físico, interações sociais, gestão do estresse e estratégias de enfrentamento, prevenção de vícios. Além de treinamentos para a população e os próprios profissionais de saúde no sentido de prevenir, detectar e tratar o alerta precoce de sintomas de transtorno de estresse pós-traumático. ${ }^{48}$

No artigo ${ }^{47}$ ele vai reforçar o papel dos profissionais de saúde como: educação do público sobre os efeitos psicológicos comuns de uma pandemia; motivar o público a adotar estratégias para prevenção de doenças e promoção da saúde; integrar os serviços de saúde mental aos serviços de saúde disponíveis; ensinar estratégias de solução de problemas para lidar com a crise atual; capacitar os pacientes com COVID-19 e seus cuidadores; e fornecer assistência em saúde mental aos profissionais de saúde.

O artigo ainda aponta a necessidade de desenvolvimento de equipes de especialistas qualificados para lidar com o estresse emocional; o treinamento dos profissionais de saúde comunitária em aspectos básicos dos cuidados de saúde mental; o uso de pesquisas on-line para avaliar o escopo dos problemas de saúde mental; o desenvolvimento de materiais on-line para educação em saúde mental; a prestação de serviços de aconselhamento e autoajuda on-line; o desenvolvimento de serviços de telemedicina para fins de diagnóstico e aconselhamento; e a necessidade de tornar os serviços de saúde mental on-line acessíveis a indivíduos de camadas socioeconômicas mais baixas. ${ }^{47}$

Medidas semelhantes também foram adotadas pelo Governo Indiano, incluindo medidas para reduzir a tensão financeira. Foi elaborado uma cartilha educativa para a população contendo medidas individuais para a prevenção da ansiedade, lidar com a solidão, dificuldade em se concentrar, estresse, desespero, pânico e medo, durante o período de quarentena e Lockdown no país. ${ }^{46}$

$\mathrm{O}$ artigo $^{49}$ traz a importância do uso de animais de estimação como forma de fornecer um recurso inestimável de conforto, contato físico, motivação para atividades e conexão com outras pessoas. Porém tal estudo carece de mais evidências científicas.

É importante ressaltar também as mudanças que os serviços de saúde mental da China tiveram que passar para atender a população em sofrimento mental durante a 
pandemia. 0 artigo ${ }^{42}$ relata que os pacientes internados, especialmente aqueles que necessitavam de hospitalização prolongada em enfermarias fechadas tiveram suas rotinas modificadas.

Nesse sentido as ações foram implementadas pelo governo como: as instituições de saúde mental tiveram de abordar a prevenção de infecções nosocomiais; os departamentos de saúde tiveram de fornecer tratamento e atendimento oportuno aos pacientes com problemas mentais infectados com COVID-19; e a unidade integrada de gerenciamento de saúde mental em nível de município/subdistrito tinham que fornecer gerenciamento, tratamento e atendimento comunitário aos pacientes em sofrimento mental em casa. ${ }^{42}$

Os hospitais psiquiátricos tiveram que reduzir as consultas ambulatoriais, restringir os critérios de admissão e diminuir o tempo de internação. Para pacientes psiquiátricos recém-admitidos, as enfermarias de isolamento deviam ser montadas e a visita, suspensa para minimizar o risco potencial de infecção hospitalar. Foi oferecido aconselhamento psicológico e orientação profissional em hospitais para aqueles em estados psicológicos mais graves, utilizando as medidas de proteção necessárias. Para os demais casos, os serviços on-line de saúde mental foram amplamente adotados, como linhas diretas e plataformas de aplicativos móveis. ${ }^{42}$

A consultoria on-line e equipes de resposta a crises foram designadas para fornecer educação em saúde mental para pacientes e equipe médica da linha de frente. Foi fornecido aconselhamento psicológico via hotline, WeChat e vídeo para pessoas isoladas e, foi adotada estratégias para aliviar o estresse através de mídias sociais. ${ }^{42}$

Os artigos ${ }^{42,44}$ também fazem uma crítica aos sistemas de teleatendimento psicológico, visto que políticas unificadas de gestão e coordenação ainda são inadequadas no país, o que resultou em distribuição desigual e desperdício de recursos médicos, sem falar na avaliação da eficácia desses serviços, porque faltavam dados do histórico médico, dados psicométricos, linguagem corporal e observação de acompanhamento eficaz. Algumas pessoas (por exemplo, adultos mais velhos) tinham acesso limitado a smartphones e à internet de banda larga, não se beneficiando dos serviços. E acrescenta-se o fato de profissionais de saúde, que são mais afetados psicologicamente, não encontrarem disposição após horas de plantão para acessar os serviços remotos.

Desse modo, medidas globais de saúde devem ser empregadas para lidar com estressores psicossociais, particularmente relacionados ao estigma, uso de isolamento/quarentena, medo e vulnerabilidade entre a população. Uma resposta à pandemia em todo o mundo deve incluir um foco no impacto na saúde mental dos pacientes e da população em geral. ${ }^{45}$

A principal limitação do estudo diz respeito a quantidade de artigos publicados com maior evidência ainda serem escassos, o que exige estudos clínicos mais aprofundados e que demorariam mais tempo. Em contrapartida, muitos artigos preprints, sem avaliação por pares, de qualidade questionável disponíveis online podem divulgar informações errôneas sobre o tema. Além disso, a COVID-19 ainda não é um descritor controlado, o que dificulta e limita as buscas. Aponta-se também a lacuna na produção nacional que precisa divulgar cientificamente seus achados na área (ainda restrito a artigos reflexivos), visto que as experiencias de saúde mental na China podem não refletir o contexto nacional. Outra importante lacuna a ser citada é a saúde mental de grupos vulneráveis e marginalizados da sociedade, exigindo pesquisas mais robustas com essas populações.

\section{Conclusão}

Esta scoping review atingiu os objetivos ao mapear publicações sobre os aspectos de saúde mental na população devido a pandemia por COVID-19. Desse modo, se faz imprescindível a preocupação com os impactos na saúde mental da população durante a pandemia por COVID19 , exigindo estratégias para enfrentamento desses problemas.

Pensando em se prevenir de pandemias posteriores é necessário garantir que a saúde mental seja incluída no desenho dos planos de emergência através de uma equipe multiprofissional de saúde mental. Esse contexto reforça a necessidade do fortalecimento do Sistema Único de Saúde como uma política pública capaz de responder às demandas de saúde, física e mentais, durante o período de pandemia.

É preciso desenvolver ações de saúde mental integrais, equânimes e universais, que contemplem populações vulneráveis, conhecendo a realidade de saúde mental de crianças e adolescentes, pessoas em sofrimento mental, em situação de rua, na comunidade negra, pessoas LGBTQ+, pessoas em áreas remotas e/ou rurais que enfrentam barreiras no acesso aos cuidados de saúde e aqueles pertencentes a níveis socioeconômicos mais baixos.

Além disso é preciso treinamento dos profissionais de saúde, voluntários, gestores, para que consigam contemplar aspectos da saúde mental para além dos sintomas físicos da COVID-19. Para a enfermagem, é importante pensar estratégias de cuidado à saúde mental dos sujeitos durante e pós pandemia. Vale destacar a necessidade de atenção e cuidado aos profissionais de saúde, em especial enfermeiras, visto que são as trabalhadoras da linha de frente da pandemia que mais são acometidas por problemas de saúde mental. Assim, alertase aos gestores para que se efetuem estratégias de cuidado aos trabalhadores que cuidam da população.

Sugere-se que uma vez desenvolvidas, essas intervenções devem ser testadas, para que as informações sobre estratégias terapêuticas efetivas possam ser amplamente divulgadas entre os que trabalham nesse campo. Além disso sugere-se pesquisas colaborativas, multicêntricas e interdisciplinares (enfermagem, biologia, psicologia, psiquiatria, política social, métodos qualitativos e quantitativos), a fim de aprofundar a compreensão das implicações para a saúde da crise atual. 


\section{Referências}

1. Oliveira AC, Lucas TC, Iquiapaza RA. What has the COVID-19 pandemic taught us about adopting preventive measures? Texto contexto enferm. [Internet]. 2020 [cited 2020 Ago 06];29:e20200106. Available from: https://doi.org/10.1590/1980-265x-tce-2020-0106

2. Zhu N, Zhang D, Wang W, Xingwang Li, Yang B, Song J, et al. A novel Coronavirus from patients with pneumonia in China, 2019. N Eng J Med. [Internet] 2020 [cited 2020 Apr 25]:1-7. Available from: https://doi.org/10.1056/NEJMoa2001017

3. Zhang J, Wu W, Zhao X, Zhang W. Recommended psychological crisis intervention response to the 2019 novel coronavirus pneumonia outbreak in China: a model of West China Hospital. Precision Clinical Medicine [Internet]. 2020 [cited 2020 Apr 25];3(1):3-8. Available from: https://doi.org/10.1093/pcmedi/pbaa006

4. Peters MDJ, Godfrey C, McInerney P, Munn Z, Tricco AC, Khalil, H. Chapter 11: Scoping Reviews (2020 version). In: Aromataris E, Munn Z (Editors). Joanna Briggs Institute Reviewer's Manual, JBI [Internet]. 2020 [cited 2020 Mar 30]. Available from https://reviewersmanual.joannabriggs.org/

5. Liu N, Zhang F, Wei C, Jia Y, Shang Z, Sun L, et al. Prevalence and predictors of PTSS during COVID-19 outbreak in China hardest hit areas: Gender differences matter. Psychiatry Res. [Internet]. 2020 [cited 2020 Apr 25];287:112921. Available from: https://doi.org/10.1016/j.psychres.2020.112921

6. Brooks SK, Webster RK, Smith LE, Woodland L, Wessely S, Greenberg N, et al. The psychological impact of quarantine and how to reduce it: rapid review of the evidence. Lancet [Internet]. 2020 [cited 2020 Apr 25];395:912-20. Available from: https://doi.org/10.1016/S01406736(20)30460-8

7. Li S, Wang Y, Xue J, Zhao N, Zhu T. The Impact of COVID-19 Epidemic Declaration on Psychological Consequences: A Study on Active Weibo Users. Int J Environ Res Public Health [Internet]. 2020 [cited Apr 25];17(6):e2032. Available from: https://doi.org/10.3390/ijerph17062032

8. Ahmed MZ, Ahmed O, Aibao Z, Hanbin S, Siyu L, Ahmad A. Epidemic of COVID-19 in China and associated Psychological Problems. Asian J Psychiatr [Internet]. 2020 [cited 2020 Apr 25];51:e102092. Available from: https://doi.org/10.1016/j.ajp.2020.102092

9. Wang C, Pan R, Wan X, Tan Y, Xu L, Mclntyre RS, et al. A longitudinal study on the mental health of general population during the COVID19 epidemic in China. Brain Behav Immun [Internet]. 2020 [cited 2020 Apr 25];87:40-8. Available from: https://doi.org/10.1016/j.bbi.2020.04.028

10. Moghanibashi-Mansourieh A. Assessing the anxiety level of Iranian general population during COVID-19 outbreak. Asian J Psychiatr [Internet]. 2020 [cited 2020 Apr 25];51:e102076. Available from: https://doi.org/10.1016/j.ajp.2020.102076

11. Lee SA. Coronavirus anxiety scale: A brief mental health screener for COVID-19 related anxiety. Death Stud [Internet]. 2020 [cited 2020 Apr 25];44(7):393-401. Available from: https://doi.org/10.1080/07481187.2020.1748481

12. Huang Y, Zhao N. Generalized anxiety disorder, depressive symptoms and sleep quality during COVID-19 outbreak in China: a web-based cross-sectional survey. Psychiatry Res [Internet]. 2020 [cited 2020 Apr 26];12(288):e112954. Available from: https://doi.org/10.1016/j.psychres.2020.112954

13. Tian F, Li H, Tian S, Yang J, Shao J, Tian C. Psychological symptoms of ordinary Chinese citizens based on SCL-90 during the level I emergency response to COVID-19. Psychiatry Res [Internet]. 2020 [cited 2020 Apr 26];11(288):e112992. Available from: https://doi.org/10.1016/j.psychres.2020.112992

14. Wang Y, Di Y, Ye J, Wei W. Study on the public psychological states and its related factors during the outbreak of coronavirus disease 2019 (COVID-19) in some regions of China. Psychol Health Med [Internet]. 2020 [cited 2020 Apr 26];30:1-10. Available from: https://doi.org/10.1080/13548506.2020.1746817

15. Nguyen HC, Nguyen MH, Do BN, Tran CQ, Nguyen TTP, Pham KM, et al. People with Suspected COVID-19 Symptoms Were More Likely Depressed and Had Lower Health-Related Quality of Life: The Potential Benefit of Health Literacy. J Clin Med [Internet]. 2020 [cited 2020 Apr 26];9(4):e965. Available from: https://doi.org/10.3390/jcm9040965

16. Xiao H, Zhang Y, Kong D, Li S, Yang N. Social Capital and Sleep Quality in Individuals Who Self-Isolated for 14 Days During the Coronavirus Disease 2019 (COVID-19) Outbreak in January 2020 in China. Med Sci Monit [Internet]. 2020 [cited 2020 Apr 26];26:e923921. Available from: https://doi.org/10.12659/MSM.923921

17. Roy D, Tripathy S, Kar SK, Sharma N, Verma SK, Kaushal V. Study of knowledge, attitude, anxiety \& perceived mental healthcare need in Indian population during COVID-19 pandemic. Asian J Psychiatr [Internet]. 2020 [cited 2020 Apr 26];51:102083. Available from: https://doi.org/10.1016/j.ajp.2020.102083

18. Wang C, Pan R, Wan X, Tan Y, Xu L, Ho CS, et al. Immediate Psychological Responses and Associated Factors during the Initial Stage of the 2019 Coronavirus Disease (COVID-19) Epidemic among the General Population in China. Int J Environ Res Public Health [Internet]. 2020 [cited 2020 Apr 26];17(5):e1729. Available from: https://doi.org/10.3390/ijerph17051729

19. Zhang Y, Ma ZF. Impact of the COVID-19 Pandemic on Mental Health and Quality of Life among Local Residents in Liaoning Province, China: A Cross-Sectional Study. Int J Environ Res Public Health [Internet]. 2020 [cited 2020 Apr 26];17(7):e2381. Available from: https://doi.org/10.3390/ijerph17072381

20. Mejia CR, Rodriguez-Alarcon JF, Garay-Rios L, Enriquez-Anco MG, Moreno A, Huaytan-Rojas K, et al. Percepción de miedo o exageración que transmiten los medios de comunicación en la población peruana durante la pandemia de la COVID-19. Rev. cuban invest. bioméd [Internet]. 2020 [cited 2020 May 7];39(2):e698. Available from: http://www.revibiomedica.sld.cu/index.php/ibi/article/view/698

21. Gao J, Zheng P, Jia Y, Chen H, Mao Y, Chen S, et al. Mental health problems and social media exposure during COVID-19 outbreak. PLoS One [Internet]. 2020 [cited 2020 Apr 26];15(4):e0231924. Available from: https://doi.org/10.1371/journal.pone.0231924

22. Sønderskov KM, Dinesen PT, Santini ZI, Østergaar SD. The Depressive State of Denmark during the COVID-19 Pandemic. Acta Neuropsychiatr [Internet]. 2020 [cited 2020 Apr 26];22:1-3. Available from: https://doi.org/10.1017/neu.2020.15

23. Cao W, Fang Z, Hou G, Han M, Xu X, Dong J, et al. The psychological impact of the COVID-19 epidemic on college students in China. Psychiatry Res [Internet]. 2020 [cited 2020 Apr 28];287:112934. Available from: https://doi.org/10.1016/j.psychres.2020.112934 
24. Xiang YT, Zhao YJ, Liu ZH, Li XH, Zhao N, Cheung T, et al. The COVID-19 outbreak and psychiatric hospitals in China: managing challenges through mental health service reform. Int J Biol Sci [Internet]. 2020 [cited 2020 Apr 28];16(10):1741-4. Available from:

https://doi.org/10.7150/ijbs.45072

25. Zhang J, Shuai L, Yu H, Wang Z, Qiu M, Lu L, et al. Acute stress, behavioural symptoms and mood states among school-age children with attention-deficit/hyperactive disorder during the COVID-19 outbreak. Asian J Psychiatr [Internet]. 2020 [cited 2020 Apr 28];51:e102077. Available from: https://doi.org/10.1016/j.ajp.2020.102077

26. Yang $\mathrm{H}, \mathrm{Bin} \mathrm{P}, \mathrm{He}$ AJ. Opinions from the epicenter: an online survey of university students in Wuhan amidst the COVID-19 outbreak, Journal of Chinese Governance [Internet]. 2020 [cited 2020 Apr 28]. Available from: https://doi.org/10.1080/23812346.2020.1745411

27. Zhang SX, Wang Y, Rauch A, Wei F. Unprecedented disruption of lives and work: Health, distress and life satisfaction of working adults in China one month into the COVID-19 outbreak. Psychiatry Res [Internet]. 2020 [cited 2020 Apr 28];288:e112958. Available from: https://doi.org/10.1016/j.psychres.2020.112958

28. Phillips P, Titterton A, Tooma M. Managing The health and safety legal and psychological ramifications of COVID-19: an Australian multidisciplinary perspective. SR [Internet]. 2020 [cited 2020 May 7];4(3):45-6. Available from: https://pressto.amu.edu.pl/index.php/sr/article/view/22534

29. Sá-Serafim R, Bú ED; Lima-Nunes A. Manual de Diretrizes para Atenção Psicológica nos Hospitais em Tempos de Combate ao COVID-19. Rev. Saúde Ciênc. Online [Internet]. 2020 [cited 2020 May 7];8(2)S2:1-24. Available from: http://www.ufcg.edu.br/revistasaudeeciencia/index.php/RSC-UFCG/article/view/876/456

30. Greenberg N, Docherty M, Gnanapragasam S, Wessely S. Managing mental health challenges faced by healthcare workers during covid19 pandemic. BMJ [Internet]. 2020 [cited 2020 May 7];368:m1211. Available from: https://doi.org/10.1136/bmj.m1211

31. Lai J, Ma S, Wang Y, Cai Z, Hu J, Wei N, et al. Factors Associated With Mental Health Outcomes Among Health Care Workers Exposed to Coronavirus Disease 2019. JAMA Netw Open [Internet]. 2020 [cited 2020 May 7];3(3):e203976. Available from: https://doi.org/10.1001/jamanetworkopen.2020.3976

32. Chew NWS, Lee GKH, Tan BYQ, Jing M, Goh Y, Ngiam NJH, et al. A multinational, multicentre study on the psychological outcomes and associated physical symptoms amongst healthcare workers during COVID-19 outbreak. Brain Behav Immun [Internet]. 2020 [cited 2020 May 7];S0889-1591(20)30523-7. Available from: https://doi.org/10.1016/j.bbi.2020.04.049

33. Sun N, Shi S, Jiao D, Song R, Ma L, Wang H, et al. A Qualitative Study on the Psychological Experience of Caregivers of COVID-19 Patients. Am J Infect Control [Internet]. 2020 [cited 2020 Apr 28];48:592-8. Available from: https://doi.org/10.1016/j.ajic.2020.03.018

34. Yin X, Zeng L. A study on the psychological needs of nurses caring for patients with coronavirus disease 2019 from the perspective of the existence, relatedness, and growth theory. Int J Nurs Sci [Internet]. 2020 [cited 2020 Apr 28];7(2):157-60. Available from: https://doi.org/10.1016/j.ijnss.2020.04.002

35. Kanga L, Ma S, Chen M, Yang J, Wang Y, Li R, et al. Impact on mental health and perceptions of psychological care among medical and nursing staff in Wuhan during the 2019 novel coronavirus disease outbreak: A cross-sectional study. Brain Behav Immun [Internet]. 2020 [cited 2020 May 7];S0889-1591(20)30348-2. Available from: https://doi.org/10.1016/j.bbi.2020.03.028

36. Zhang W, Wang K, Yin L, Zhao W, Xue Q, Peng M, et al. Mental Health and Psychosocial Problems of Medical Health Workers during the COVID-19 Epidemic in China. Psychother Psychosom [Internet]. 2020 [cited 2020 May 7];9:1-9. Available from: https://doi.org/10.1159/000507639

37. Lu W, Wang H, Lin Y, Li L. Psychological status of medical workforce during the COVID-19 pandemic: A cross-sectional study. Psychiatry Res [Internet]. 2020 [cited 2020 May 7];288:e112936. Available from: https://doi.org/10.1016/j.psychres.2020.112936

38. Xiao H, Zhang Y, Kong D, Li S, Yang N. The Effects of Social Support on Sleep Quality of Medical Staff Treating Patients with Coronavirus Disease 2019 (COVID-19) in January and February 2020 in China. Med Sci Monit [Internet]. 2020 [cited 2020 May 7];26:e923549. Available from: https://doi.org/10.12659/MSM.923549

39. Shah K, Kamrai D, Mekala H, Mann B, Desai K, Pate RS. Focus on Mental Health During the Coronavirus (COVID-19) Pandemic: Applying Learnings from the Past Outbreaks. Cureus [Internet]. 2020 [cited 2020 May 7];12(3):e7405. Available from: https://doi.org/10.7759/cureus.7405

40. Casale S, Flett GL. Interpersonally based fears during the COVID-19 pandemic: Reflections on the fear of missing out and the fear of not mattering constructs. Clinical Neuropsychiatry [Internet]. 2020 [cited 2020 May 10];17(2):88-93. Available from: https://doi.org/10.36131/CN20200211

41. Mo Y, Deng L, Zhang L, Lang Q, Liao C, Wang N, et al. Work stress among Chinese nurses to support Wuhan for fighting against the COVID19 epidemic. J Nurs Manag [Internet]. 2020 [cited 2020 Apr 28];28:1002-9. Available from: https://doi.org/10.1111/jonm.13014

42. Li W, Yang Y, Liu ZH, Zhao YJ, Zhang Q, Zhang L, et al. Progression of Mental Health Services during the COVID-19 Outbreak in China. Int J Biol Sci [Internet]. 2020 [cited 2020 May 10];16(10):1732-8. Available from: https://doi.org/10.7150/ijbs.45120

43. Medeiros A, Pereira E, Silva R, Dias F. Fases psicológicas e sentido da vida em tempos de isolamento social por pandemia COVID-19 uma reflexão a luz de Viktor Frankl. Research, Society and Development [Internet]. 2020 [cited 2020 Apr 28];9(5):e122953331. Available from: https://doi.org/10.33448/rsd-v9i5.3331

44. Jiang X, Deng L, Zhu Y, Ji H, Tao L, Liu L, et al. Psychological crisis intervention during the outbreak period of new coronavirus pneumonia from experience in Shanghai. Psychiatry Res [Internet]. 2020 [cited 2020 May 10];286:112903. Available from: https://doi.org/10.1016/j.psychres.2020.112903

45. Torales J, O'Higgins M, Castaldelli-Maia JM, Ventriglio A. The outbreak of COVID-19 coronavirus and its impact on global mental health. Int J Soc Psychiatry [Internet]. 2020 [cited 2020 May 10];31:20764020915212. Available from: https://doi.org/10.1177/0020764020915212

46. Hiremath P, Kowshik CSS, Manjunath M, Shettar M. COVID 19: Impact of Lock-down on Mental Health and Tips to Overcome. Asian J Psychiatr [Internet]. 2020 [cited 2020 May 10];10:e102088. Available from: https://doi.org/10.1016/j.ajp.2020.102088

47. Rajkumar RP. COVID-19 and mental health: A review of the existing literature. Asian J Psychiatr [Internet]. 2020 [cited 2020 May 10];52:e102066. Available from: https://doi.org/10.1016/j.ajp.2020.102066 
48. Chevance A, Gourion D, Hoertel N, Llorca PM, Thomas P, Bocher R, et al. Ensuring mental health care during the SARS-CoV-2 epidemic in France: A narrative review. Encephale [Internet]. 2020 [cited 2020 May 10];46(3supp):3-13. Available from: https://doi.org/10.1016/j.encep.2020.03.001

49. Hoy-Gerlach J, Rauktis M, Newhill C. (Non-human) animal companionship: a crucial support for people during the covid-19 pandemic. SR [Internet]. 2020 [cited 2020 May 7];4(2):109-20. Available from: https://pressto.amu.edu.pl/index.php/sr/article/view/22511

50. Ren SY, Gao RD, Chen YL. Fear can be more harmful than the severe acute respiratory syndrome coronavirus 2 in controlling the corona virus disease 2019 epidemic. World J Clin Cases [Internet]. 2020 [cited 2020 May 15];8(4):652-7. Available from: https://doi.org/10.12998/wjcc.v8.i4.652

51. Yi Y, Lagniton PNP, Ye S, Li E, Xu RH. COVID-19: what has been learned and to be learned about the novel coronavirus disease. Int J Biol Sci [Internet]. 2020 [cited 2020 May 15];16(10):1753-66. Available from: https://doi.org/10.7150/ijbs.45134

52. Horesh D, Brown AD. Traumatic Stress in the Age of COVID-19: A Call to Close Critical Gaps and Adapt to New Realities. Psychol Trauma [Internet]. 2020 [cited 2020 May 10];12(4):331-5. Available from: https://doi.org/10.1037/tra0000592 